\title{
Study on the protective effect of Lycopene on ischemia-reperfusion myocardium through Inhibiting the opening of mitochondrial MPTP and the activation of apoptotic pathway
}

\author{
Juan WANG ${ }^{1 \#}$, Jianglin FU ${ }^{1 \#}$, Dan CHEN ${ }^{1 *}$
}

\begin{abstract}
Through in vitro experiments, the paper investigated whether Lycopene can mitigate cardiac ischemia/reperfusion injury, and further explored whether the underlying mechanism works by inhibiting the opening of MPTP.A hypoxia-reoxygenation model was established. CCK8 was employed to determine the viability of $\mathrm{H} 9 \mathrm{c} 2$ cardiomyocytes. $\mathrm{LDH}$ was used to determine the degree of damage of H9c2 cardiomyocytes. Trypan blue staining was applied to determine the survival rate of H9c2 cardiomyocytes. Flow cytometry was used to determine the apoptosis rate of $\mathrm{H} 9 \mathrm{c} 2$ cardiomyocytes. Rhodamine 123 staining method was applied to evaluate the changes of mitochondrial membrane potential of H9c2 cardiomyocytes. Western blot was employed to determine the protein expressions of related factors of MPTP downstream apoptosis pathway in H9c2 cardiomyocytes. Calcium induction method was used to evaluate the opening degree of MPTP of H9c2 cardiomyocytes. Western blot was applied to determine the protein expressions of the related factors regulating MPTP opening in $\mathrm{H} 9 \mathrm{c} 2$ cardiomyocytes. The vitality of $\mathrm{H} 9 \mathrm{c} 2$ cardiomyocytes in the Lycopene group was significantly improved, the LDH activity was significantly reduced, and the cell survival rate was significantly improved. The apoptosis rate was significantly reduced, and the membrane potential decreased significantly. The expression levels of Bax, cytochrome C, APAF-1, caspase 9 and caspase-3 proteins were significantly reduced. The sensitivity of MPTP opening was significantly decreased. Lycopene can effectively alleviate the hypoxia/reoxygenation injury of H9c2 cardiomyocytes. By up-regulating Bcl-2 and down-regulating Bax, Lycopene can inhibit the opening of mitochondrial MPTP and the activation of downstream apoptotic pathways, thereby reducing apoptosis.
\end{abstract}

Keywords: Lycopene; ischemia-reperfusion; MPTP; apoptosis.

Practical Application: Lycopene on Ischemia-reperfusion Myocardium.

\section{Introduction}

The heart is the most energy-consuming organ in the human body, but the concentration of ATP in myocardial cells is relatively low, which means that myocardial cells must continuously synthesize ATP to maintain normal diastolic and contractile functions. Mitochondria are the most important place for intracellular oxidative phosphorylation and ATP synthesis. Cardiomyocytes contain a large number of mitochondria, a double-layer membrane-coated organelle that exists in most cells. It is the main structure for energy production in cells and the main place for cells to carry out aerobic respiration. In addition, mitochondria are also involved in cell apoptosis and signal transduction, cell proliferation, cell metabolism and other processes. Mitochondrial injury is the main pathogenesis that causes myocardial ischemia/reperfusion injury, which in turn leads to myocardial death, apoptosis and contractile failure. Therefore, mitochondrial dysfunction is a determinant of myocardial ischemia/reperfusion injury. Studies have found that autologous mitochondria transplantation, in which a person's autologous healthy mitochondria is delivered to the ischemic area of damaged mitochondria through mitochondria in coronary arteries, can significantly improve myocardial function
(Giorgio et al., 2018; Shin et al., 2019; Chiva-Blanch \& Badimon, 2017; Espinosa-Moncada et al., 2018; Vetrani et al., 2018).

Lycopene has a long-chain polyunsaturated olefin molecular structure, which gives it a strong capability to eliminate free radicals and antioxidant capacity. The current research on its biological effects mainly focuses on anti-oxidation, reducing the risk of cardiovascular disease, reducing genetic damage, and inhibiting the occurrence and development of tumors. Our research is to explore whether Lycopene can mitigate cardiac ischemia/reperfusion injury, and to further explore whether its underlying mechanism plays a role by inhibiting the opening of MPTP.

\section{Methods}

\subsection{Cell grouping and CCK-8 to detect the viability of $\mathrm{H} 9 \mathrm{c} 2$ cells}

H9c2 cells were obtained. Cell viability was determined according to the operation manual, and the OD value at $450 \mathrm{~nm}$ was measured. The groupings are as follows: A, the Control group,

\footnotetext{
"These authors contributed equally to this work.

Received 05 June, 2021

Accepted 14 June, 2021

${ }^{1}$ Department of Cardiovascular Medicine, Shiyan Renmin Hospital, Hubei University of Medicine, Hubei, China

*Corresponding author: chendanswe5@sina.com
} 
in which the cells were grown in an incubator, without receiving sugar-free Earle's medium replacement; B, the H/R group, in which the cells received sugar-free Earle's medium and normal medium in sequence, meaning that the cells were treated with hypoxia for $12 \mathrm{~h}$ and reoxygenation for $1 \mathrm{~h}$ respectively; $\mathrm{C}$, the low-dose drug group. After pretreatment with $5 \mu \mathrm{M}$ Lycopene for $4 \mathrm{~h}$, the cells received sugar-free Earle's medium and normal medium, and then received $12 \mathrm{~h}$ hypoxia and $1 \mathrm{~h}$ reoxygenation treatment; $\mathrm{D}$, the middle-dose drug group, the cells received $4 \mathrm{~h}$ pretreatment with $10 \mu \mathrm{M}$ Lycopene, other procedures are the same as group C; E, the high-dose drug group, the cells received pretreatment with $20 \mu \mathrm{M}$ Lycopene for $4 \mathrm{~h}$, with other procedures the same as group $\mathrm{C}$.

\subsection{Trypan blue staining method to determine the survival rate of $\mathrm{H} 9 \mathrm{c} 2$ cells.}

H9c2 cells were prepared. According to the operation procedure and principle of trypan blue staining: normal cardiomyocytes are not stained, only cardiomyocytes with loss of cell membrane integrity can be stained blue by trypan blue. Therefore, the calculation formula of cell survival rate is: cell survival rate $=$ total number of unstained cells/total number of cells $\times 100 \%$, and the grouping is the same as above.

\subsection{LDH activity detection of $\mathrm{H} 9 \mathrm{c} 2$ cell damage.}

Following the instructions, the activity of $\mathrm{LDH}$ was calculated according to the formula: $\mathrm{LDH}(\mathrm{U} / \mathrm{L})=($ measured $\mathrm{OD}$ value - control OD value) / (standard OD value - blank OD value) $\times$ standard concentration $\times 1000$.

\subsection{Flow cytometry to measure the apoptosis rate of $\mathrm{H} 9 \mathrm{c} 2$ cells}

The groups are as follows: A, the Control group; B, the H/R group; $C$ group, the cells were pretreated with $20 \mu \mathrm{M}$ Lycopene for $4 \mathrm{~h}$; and D group, the cells were pretreated with $20 \mu \mathrm{M}$ Lycopene $+20 \mu \mathrm{M}$ Atractylodes carboxylate for $4 \mathrm{~h}$. Flow cytometry was used for apoptosis detection.

\subsection{Rhodamine 123 method to measure the changes of mitochondrial membrane potential of H9c2 cardiomyocytes.}

According to the operation and dyeing principle of Rhodamine 123 , the stronger fluorescence intensity and denser fluorescence mean the less reduction of mitochondrial membrane potential. The weaker fluorescence intensity and sparser fluorescence suggest more reduction of mitochondrial membrane potential.

\subsection{Calcium induction method to measure the opening degree of mitochondria of $\mathrm{H} 9 \mathrm{c} 2$ cardiomyocytes.}

H9c2 cardiomyocyte mitochondria were extracted. Buffer A of the $190 \mu \mathrm{L}$ of MPTP detection kit was added to the mitochondrial pellets of each group. The microplate reader was used for continuous detection of the $\mathrm{A} 540$ from $0 \mathrm{~min}$ to $10 \mathrm{~min}$. The samples were added with Buffer B of the $10 \mu \mathrm{L}$ of MPTP detection kit. A microplate reader was used to measure once at A540 at an interval of $1 \mathrm{~min}$, and continuously for $10 \mathrm{~min}$.

\subsection{Detection of protein expression by WB method.}

After SDS-PAGE gel electrophoresis, membrane transfer, western blotting, chemiluminescence operation, the expressions of $\mathrm{Bcl}-2$, Bax, cytochrome C, APAF-1, caspase 9 and caspase- 3 proteins were determined.

\subsection{Statistical analysis.}

The data were analyzed using SPSS17.0 software. All data were expressed as mean \pm standard deviation. Multi-sample comparisons of 3 or more groups were conducted using oneway analysis of variance, and the least significant difference test was used for multiple comparison pairwise. If $\mathrm{P}<0.05$, it is considered a statistically significant difference.

\section{Result}

\subsection{The effect of Lycopene on the vitality of hypoxia/ reoxygenation $\mathrm{H} 9 \mathrm{c} 2$ cardiomyocytes.}

Compared with the Control group, the A450 value of the $H / R$ group was significantly reduced, and different concentrations of Lycopene can effectively improve the viability of H9c2 cardiomyocytes, as shown in Figure 1.

\subsection{The effect of Lycopene on the survival rate of hypoxia/ reoxygenation $\mathrm{H} 9 \mathrm{c} 2$ cardiomyocytes.}

Compared with the Control group, the survival rate of the H/R group was significantly reduced. Different concentrations of Lycopene can significantly improve the survival rate of H9c2 cardiomyocytes, as shown in Figure 2.

\subsection{The effect of Lycopene on hypoxia/reoxygenation H9c2 cardiomyocyte damage.}

Compared with the Control group, the LDH value of the H/R group was significantly higher. Different concentrations of Lycopene can effectively mitigate the damage of $\mathrm{H} 9 \mathrm{c} 2$ cardiomyocytes, as shown in Figure 3.

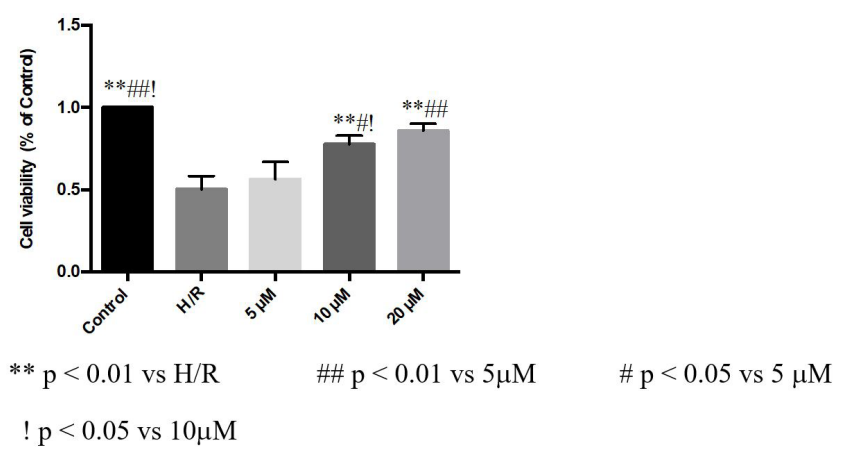

Figure 1. H9c2 myocardial cell viability results. 


\subsection{The effect of Lycopene on the apoptosis rate of hypoxia/ reoxygenation $\mathrm{H} 9 \mathrm{c} 2$ cardiomyocytes.}

Compared with the Control group, the apoptosis rate of the H/R group was significantly increased. Compared with the Lycopene group, the apoptosis rate measured in the H/R group was also significantly increased. Compared with the Control group, the protein expression level of the Lycopene + Atractylodes carboxylate group was significantly increased, as shown in Figure 4.

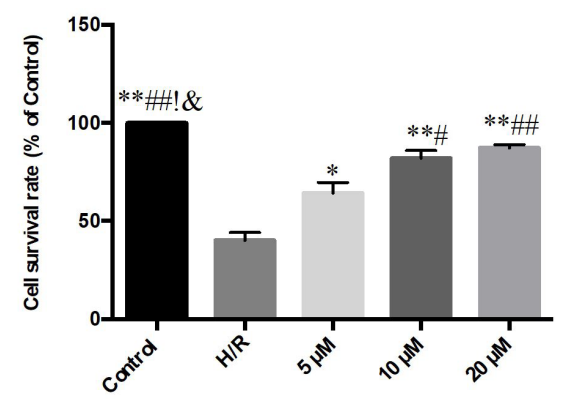

$$
\begin{aligned}
& \text { ** } \mathrm{p}<0.01 \text { vs H/R } \quad * \mathrm{p}<0.05 \text { vs H/R \# } \mathrm{p}<0.01 \text { vs } 5 \mu \mathrm{M} \quad \# \mathrm{p}< \\
& 0.05 \text { vs } 5 \mu \mathrm{M} \quad ! \mathrm{p}<0.05 \text { vs } 10 \mu \mathrm{M} \quad \& \quad \mathrm{p}<0.05 \text { vs } 20 \mu \mathrm{M}
\end{aligned}
$$

Figure 2. Detection of $\mathrm{H} 9 \mathrm{c} 2$ cardiomyocyte viability.

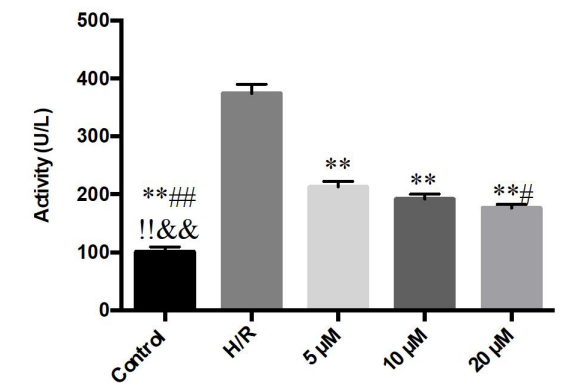

$$
\begin{array}{lrr}
{ }^{* *} \text { p }<0.01 \text { vs H/R } & \text { \#\# } \text { p }<0.01 \text { vs } 5 \mu \mathrm{M} & \text { \# p }<0.05 \text { vs } 5 \mu \mathrm{M}
\end{array} \quad \text { !! p }
$$

Figure 3. Detection of $\mathrm{H} 9 \mathrm{c} 2$ cardiomyocyte damage.

\subsection{The effect of Lycopene on the changes of mitochondrial membrane potential of hypoxia/reoxygenation $\mathrm{H} 9 \mathrm{c} 2$ cardiomyocytes.}

Rhodamine 123 is a fluorescent dye that can penetrate the mitochondrial membrane. The amount of Rhodamine 123 entering the mitochondria is indeed closely related to the level of the mitochondrial membrane potential. If the mitochondrial membrane potential is high, more Rhodamine 123 will enter and retain, with strong fluorescence, and vice versa. Therefore, the fluorescence intensity of Rhodamine 123 reflected the level of mitochondrial membrane potential. Compared with the Control group, the mitochondria $\Delta \Psi \mathrm{m}$ of the $\mathrm{H} / \mathrm{R}$ group was significantly reduced. Compared with the Lycopene group, the mitochondria $\Delta \Psi \mathrm{m}$ of the $\mathrm{H} / \mathrm{R}$ group was significantly reduced, and the mitochondria $\Delta \Psi \mathrm{m}$ of the Lycopene + Atractylodes carboxylate group was also significantly reduced, as shown in Figure 5.

\subsection{The effect of Lycopene on the expression of pro-apoptotic factor protein in the mitochondrial apoptosis pathway.}

Compared with the Control group, the protein expression level of the H/R group was significantly higher. Compared with the Lycopene group, the protein expression level of the H/R group was significantly increased, and the protein expression level of Lycopene + Atractylodes carboxylate was also significantly increased, as shown in Figure 6.

Compared with the Control group, the Bcl-2 expression of the $\mathrm{H} / \mathrm{R}$ group was significantly reduced, the Bax expression was significantly increased, thus the $\mathrm{Bcl}-2 / \mathrm{Bax}$ protein ratio was greatly reduced. Compared with the Lycopene group, the expression level of $\mathrm{Bcl}-2$ in the $\mathrm{H} / \mathrm{R}$ group was significantly reduced, the expression level of Bax was significantly increased, so the ratio of Bcl-2/Bax was obviously reduced. The Bcl-2 expression level of the Lycopene + Atractylodes carboxylate group was significantly reduced, and the Bax expression level was also significantly increased, as shown in Figure 7.
A

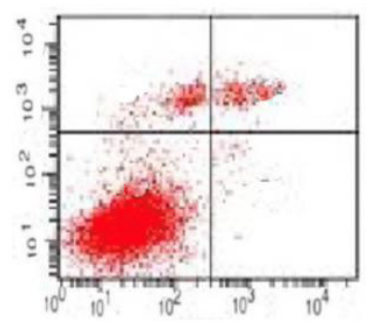

B

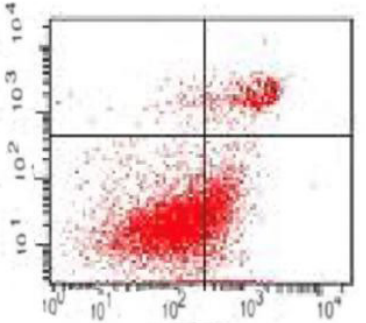

C

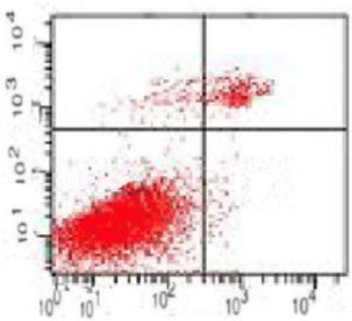

D

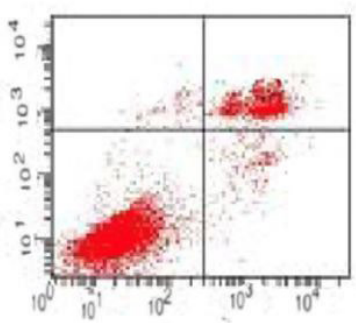

\section{A: Control \\ B: $H / R$ \\ C: $20 \mu \mathrm{M}$ Lycopene \\ D: $20 \mu \mathrm{M}$ Lycopene +20}

\section{$\mu \mathrm{M}$ Atractylodes carboxylate}

Figure 4. Detection of $\mathrm{H} 9 \mathrm{c} 2$ cardiomyocyte apoptosis. 
A

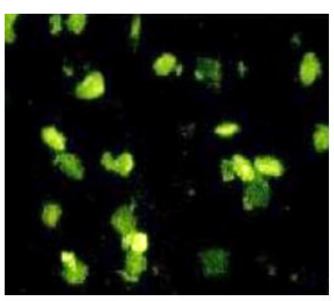

B

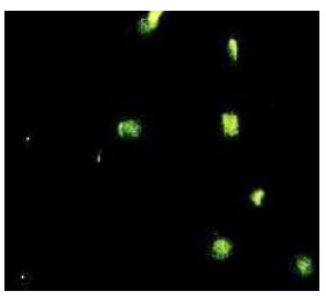

$\mathrm{C}$

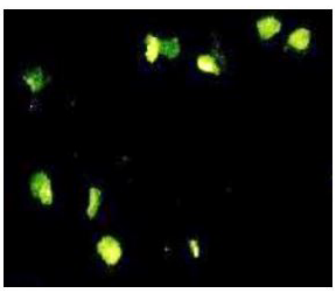

$\mathrm{D}$

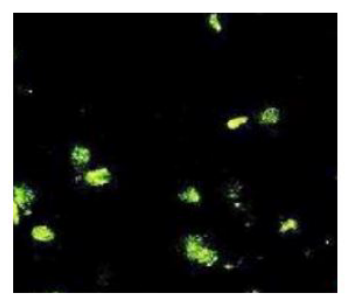

A: Control

B: $\mathrm{H} / \mathrm{R}$

C: $20 \mu \mathrm{M}$ Lycopene

D: $20 \mu \mathrm{M}$ Lycopene +20

\section{$\mu \mathrm{M}$ Atractylodes carboxylate}

Figure 5. Changes of $\Delta \Psi \mathrm{m}$ in mitochondria of $\mathrm{H} 9 \mathrm{c} 2$ cardiomyocytes.

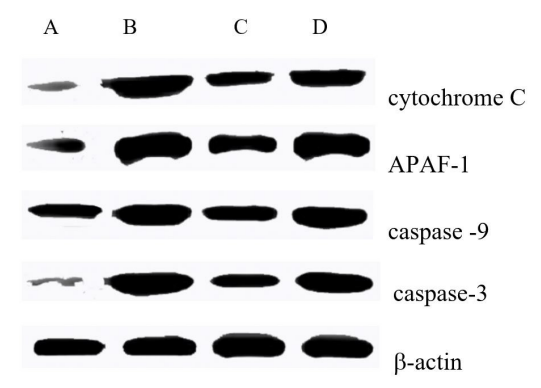

$\begin{array}{lll}\text { A: Control } & \text { B: } H / R & \text { C: } 20 \mu \mathrm{M} \text { Lycopene }\end{array}$

$\mu \mathrm{M}$ Atractylodes carboxylate

Figure 6. The protein expressions of pro-apoptotic factors in $\mathrm{H} 9 \mathrm{c} 2$ cardiomyocytes.

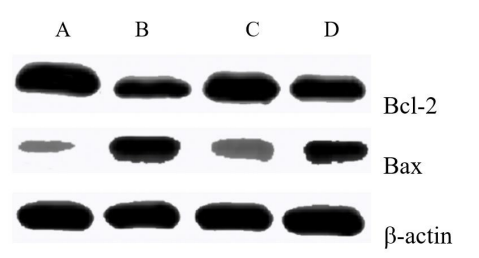

A: Control B: H/R

C: $20 \mu \mathrm{M}$ Lycopene

D: $20 \mu \mathrm{M}$ Lycopene +20

$\mu \mathrm{M}$ Atractylodes carboxylate

Figure 7. The expression levels of Bcl-2 and Bax proteins in H9c2 cardiomyocytes.

\subsection{The effect of Lycopene on the opening degree of mitochondria of hypoxia/reoxygenation H9c2 cardiomyocytes.}

The samples of each group were continuously measured with a microplate reader at the $\mathrm{A} 540$ from $0 \mathrm{~min}$ to $10 \mathrm{~min}$. The lower measured value means the higher sensitivity of MPTP to calcium, suggesting the higher openness of MPTP. The sensitivity of MPTP to calcium, that is, the opening degree of MPTP, which is reflected by the ratio of A540 (10 min) / A540 (0 min), is quantified. Because the value measured at A540 (0 min) is the highest, while the value measured at A540 (10 $\mathrm{min})$ is the lowest, the ratio is expressed as $\mathrm{min} / \mathrm{max} \mathrm{A} 540$. The lower ratio of min/max A540 suggests the higher sensitivity of MPTP to calcium and the higher opening degree of MPTP, and vice versa. Our results showed that, compared with the Control group, the A540 value and $\mathrm{min} / \mathrm{max}$ A540 value measured in the H/R group were significantly reduced. Compared with the administration group, the A540 value and min/max A540 value measured in the H/R group were significantly reduced, See Figure 8.

\section{Discussion.}

At present, substantive progress has been made in cellular events that may lead to fatal I/R injury following reperfusion after ischemia, and mitochondrial integrity is the core of this work. A number of mitochondrial-centric mechanisms are believed to play a role, such as the generation of reactive oxygen species (ROS), the opening of the mitochondrial permeability transition pore (MPTP), and the activation of endogenous apoptosis (Fan et al., 2017; Costabile et al., 2019; Brown et al., 2017).

The apoptotic pathway inherent in mitochondria is characterized by increased mitochondrial membrane permeability. This loss of mitochondrial outer membrane integrity is widely regarded as the "irreversible point" of apoptosis, and Bcl-2 family proteins (including Bax, Bak, and tBID) play an important role in this process. The translocation of DRP1 to mitochondria promotes Bax/Bak recruitment, oligomerization and pore formation at OMM, which triggers the release of cytochrome c. In addition, the recruitment of OMM by Bax/Bak is accompanied by the release of a new apoptotic factor (DDP/TIMM8a), which promotes the recruitment of DRP1 to the Bax/Bak site. DRP1 then passes through Bax/baki-dependent ubiquitination and stabilizes.

Mitochondria are the core participants in regulating cell homeostasis. They are essential for energy production. However, reactive oxygen species accumulate as by-products of the electron transport chain, causing mitochondrial damage. Oxidative stress-mediated ROS leads to rapid depolarization of 

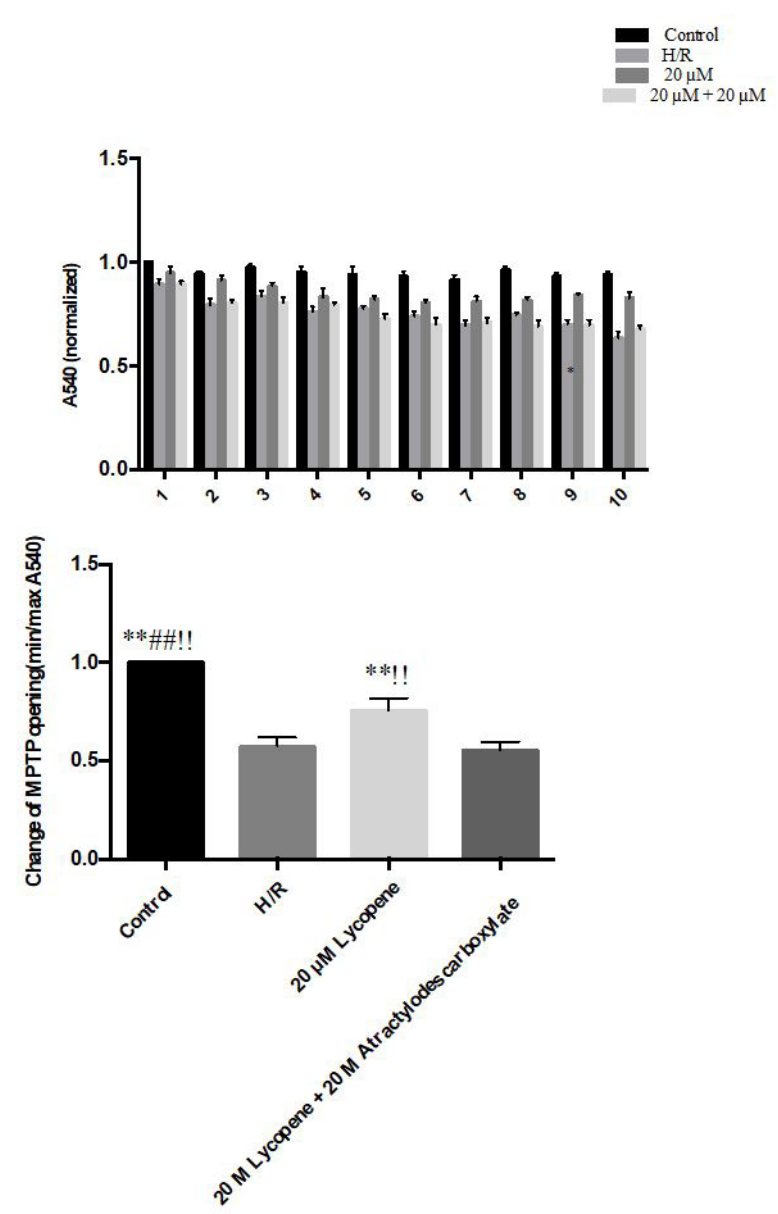

** $\mathrm{p}<0.01$ vs $\mathrm{H} / \mathrm{R} \quad$ \#\# $\mathrm{p}<0.01$ vs $20 \mu \mathrm{M} \quad$ !! $\mathrm{p}<0.01$ vs $20 \mu \mathrm{M}$ Lycopene

$+20 \mu \mathrm{M}$ Atractylodes carboxylate

Figure 8. The effect of Lycopene on the opening degree of mitochondria of hypoxia/reoxygenation H9c2 cardiomyocytes.

the mitochondrial inner membrane potential and subsequent oxidative phosphorylation damage. Damaged mitochondria produce ROS, especially in the form of superoxide anions and hydrogen peroxide, which in turn further accelerate the production of ROS. Finally, the opening of the mitochondrial permeability transition pore is a key trigger of apoptosis. Failure to maintain calcium homeostasis is not only a consequence of metabolic disorders, but also a sign of ischemia/reperfusion injury, which also plays a role in regulating mitochondrial quality control. Calcium overload can activate the proteases and phosphatases that will change the components of the electron transport chain. These enzymes can modify the components of the electron transport chain. Calcium overload can also activate the key molecule GTPase. This enzyme is related to the mitochondrial morphology and triggers the hypertrophy of the mitochondrial membrane of the ischemic myocardium, burst of ROS production, destruction of the outer mitochondrial membrane, and opening of mPTP during ischemia/reperfusion. These metabolic sequences coordinate internal and external mitochondrial membrane reorganization mainly by activating calcium-dependent phosphatase and calcineurin (Springer et al.,
2018; Sabbah et al., 2018; Collins et al., 2018; Yu et al., 2017; Coronado et al., 2018; Yu et al., 2019).

MPTP is a multi-protein giant channel complex that spans the inner and outer mitochondrial membranes, which basically allows small molecule communication between the matrix and the cytosol. The structural composition of pores is not well understood, which is a topic of ongoing debate. Preliminary studies have shown that the pore is composed of a voltage-dependent anion channel (VDAC) located in the outer mitochondrial membrane, adenine nucleotide transporter (ANT) in the inner mitochondrial membrane, and cyclophilin D (Cyp-D) in the matrix. However, subsequent gene deletion studies have shown that the formation of MPTP does not require ANT and VDAC, partly due to the presence of different subtypes of these putative pore components. Despite these observations, it is generally believed that Cyp- $D$ is the main factor in the formation of MPTP (Zhou et al., 2019; Badi et al., 2019; Medak \& Townsend, 2019).

In our research, it was found that Lycopene can inhibit the decrease of $\Delta \Psi \mathrm{m}$ and reduce the sensitivity of MPTP to $\mathrm{Ca} 2+$, thus clarifying that Lycopene exerts an anti-apoptotic mechanism by inhibiting the opening of MPTP. On this basis, it is confirmed that Lycopene can achieve the regulation of MPTP through inhibiting BAX expression and promoting Bcl-2 expression. In order to further prove that Lycopene exerts myocardial protection by acting on MPTP and inhibiting the opening of MPTP, this study applied the MPTP opening agonist: Atractylidin. The experimental results showed that Atractylidin counteracts the effect of Lycopene in inhibiting cardiomyocyte apoptosis, and it was further found that Atractylidin counteracted the protective effect of Lycopene to stabilize mitochondria $\Delta \Psi \mathrm{m}$ and reduce the sensitivity of MPTP to Ca2+.

\section{Conclusion}

In summary, it is concluded that Lycopene plays a protective role in mitigating MIRI by acting on MPTP and inhibiting the opening of MPTP.

\section{Reference}

Badi, R. M., Mostafa, D. G., Khaleel, E. F., \& Satti, H. H. (2019). Resveratrol protects against hepatic insulin resistance in a rat's model of nonalcoholic fatty liver disease by down-regulation of GPAT-1 and DGAT2 expression and inhibition of PKC membranous translocation. Clinical and Experimental Pharmacology \& Physiology, 46(6), 545555. http://dx.doi.org/10.1111/1440-1681.13074. PMid:30773673.

Brown, D. A., Perry, J. B., Allen, M. E., Sabbah, H. N., Stauffer, B. L., Shaikh, S. R., Cleland, J. G. F., Colucci, W. S., Butler, J., Voors, A. A., Anker, S. D., Pitt, B., Pieske, B., Filippatos, G., Greene, S. J. \& Gheorghiade, M. (2017). Mitochondrial function as a therapeutic target in heart failure. Nature Reviews Cardiology, 14(4), 238-250.

Chiva-Blanch, G., \& Badimon, L. (2017). Effects of polyphenol intake on metabolic syndrome: current evidences from human trials. Oxidative Medicine and Cellular Longevity, 2017, 5812401. http:// dx.doi.org/10.1155/2017/5812401.

Collins, J., Robinson, C., Danhof, H., Knetsch, C. W., van Leeuwen, H. C., Lawley, T. D., Auchtung, J. M., \& Britton, R. A. (2018). Dietary trehalose enhances virulence of epidemic Clostridium difficile. 
Nature, 553(7688), 291-294. http://dx.doi.org/10.1038/nature25178. PMid:29310122.

Coronado, M., Fajardo, G., Nguyen, K., Zhao, M., Kooiker, K., Jung, G., Hu, D. Q., Reddy, S., Sandoval, E., Stotland, A., Gottlieb, R. A., \& Bernstein, D. (2018). Physiological mitochondrial fragmentation is a normal cardiac adaptation to increased energy demand. Circulation Research, 122(2), 282-295. http://dx.doi.org/10.1161/ CIRCRESAHA.117.310725. PMid:29233845.

Costabile, G., Vitale, M., Luongo, D., Naviglio, D., Vetrani, C., Ciciola, P., Tura, A., Castello, F., Mena, P., Del Rio, D., Capaldo, B., Rivellese, A. A., Riccardi, G., \& Giacco, R. (2019). Grape pomace polyphenols improve insulin response to a standard meal in healthy individuals: a pilot study. Clinical Nutrition, 38(6), 2727-2734. http://dx.doi. org/10.1016/j.clnu.2018.11.028. PMid:30573355.

Espinosa-Moncada, J., Marín-Echeverri, C., Galvis-Pérez, Y., CiroGómez, G., Aristizábal, J. C., Blesso, C. N., Fernandez, M. L., \& Barona-Acevedo, J. (2018). Evaluation of agraz consumption on adipocytokines, inflammation, and oxidative stress markers in women with metabolic syndrome. Nutrients, 10(11), 1639. http:// dx.doi.org/10.3390/nu10111639. PMid:30400222.

Fan, F. Y., Sang, L. X., \& Jiang, M. (2017). Catechins and their therapeutic benefits to inflammatory bowel disease. Molecules, 22(3), 484. http:// dx.doi.org/10.3390/molecules22030484. PMid:28335502.

Giorgio, V., Guo, L., Bassot, C., Petronilli, V., \& Bernardi, P. (2018). Calcium and regulation of the mitochondrial permeability transition. Cell Calcium, 70, 56-63. http://dx.doi.org/10.1016/j.ceca.2017.05.004. PMid:28522037.

Medak, K. D., \& Townsend, L. K. (2019). Adding more fat to a highfat diet only exacerbates hepatic insulin resistance. The Journal of Physiology, 597(6), 1435-1436. http://dx.doi.org/10.1113/JP277632. PMid:30653266.

Sabbah, H. N., Gupta, R. C., Singh-Gupta, V., Zhang, K., \& Lanfear, D. E. (2018). Abnormalities of mitochondrial dynamics in the failing heart: normalization following long-term therapy with elamipretide.
Cardiovascular Drugs and Therapy, 32(4), 319-328. http://dx.doi. org/10.1007/s10557-018-6805-y. PMid:29951944.

Shin, B., Saeed, M. Y., Esch, J. J., Guariento, A., Blitzer, D., Moskowitzova, K., Ramirez-Barbieri, G., Orfany, A., Thedsanamoorthy, J. K., Cowan, D. B., Inkster, J. A., Snay, E. R., Staffa, S. J., Packard, A. B., Zurakowski, D., Nido, P. J., \& McCully, J. D. (2019). A novel biological strategy for myocardial protection by intracoronary delivery. JACC. Basic to Translational Science, 4(8), 871-888. http://dx.doi.org/10.1016/j. jacbts.2019.08.007. PMid:31909298.

Springer, J. E., Visavadiya, N. P., Sullivan, P. G., \& Hall, E. D. (2018). Post-injury treatment with NIM811 promotes recovery of function in adult female rats after spinal cord contusion: a dose-response study. Journal of Neurotrauma, 35(3), 492-499. http://dx.doi.org/10.1089/ neu.2017.5167. PMid:28967329.

Vetrani, C., Vitale, M., Bozzetto, L., Della Pepa, G., Cocozza, S., Costabile, G., Mangione, A., Cipriano, P., Annuzzi, G., \& Rivellese, A. A. (2018). Association between different dietary polyphenol subclasses and the improvement in cardiometabolic risk factors: evidence from a randomized controlled clinical trial. Acta Diabetologica, 55(2), 149153. http://dx.doi.org/10.1007/s00592-017-1075-x. PMid:29151225.

Yu, W., Gao, B., Li, N., Wang, J., Qiu, C., Zhang, G., Liu, M., Zhang, R., Li, C., Ji, G., \& Zhang, Y. (2017). Sirt3 deficiency exacerbates diabetic cardiac dysfunction: role of Foxo3A-Parkin-mediated mitophagy. Biochimica et Biophysica Acta, 1863(8), 1973-1983. http://dx.doi. org/10.1016/j.bbadis.2016.10.021. PMid:27794418.

Yu, W., Xu, M., Zhang, T., Zhang, Q., \& Zou, C. (2019). Mst1 promotes cardiac ischemia-reperfusion injury by inhibiting the ERK-CREB pathway and repressing FUNDC1-mediated mitophagy. The Journal of Physiological Sciences; JPS, 69(1), 113-127. http://dx.doi.org/10.1007/ s12576-018-0627-3. PMid:29961191.

Zhou, H., Wang, J., Hu, S., Zhu, H., Toanc, S., \& Ren, J. (2019). BI1 alleviates cardiac microvascular ischemia-reperfusion injury via modifying mitochondrial fission and inhibiting XO/ROS/F-actin pathways. Journal of Cellular Physiology, 234(4), 5056-5069. http:// dx.doi.org/10.1002/jcp.27308. PMid:30256421. 